\title{
Intraoperative Monitoring of the Recurrent Laryngeal Nerve during Thyroidectomy: A Standardized Approach (Part 1)
}

\author{
${ }^{1}$ Manuel C Durán Poveda, ${ }^{2}$ Gianlorenzo Dionigi, ${ }^{3}$ Antonio Sitges-Serra, ${ }^{4}$ Marcin Barczynski \\ ${ }^{5}$ Peter Angelos, ${ }^{6}$ Henning Dralle, ${ }^{7}$ Eimear Phelan, ${ }^{8}$ Gregory Randolph \\ ${ }^{1}$ Professor, Department of Endocrine Surgery, University Hospital of Fuenlabrada, Health Sciences School \\ King Juan Carlos University, Madrid, Spain \\ ${ }^{2}$ Associate Professor, Department of Surgical Sciences, Endocrine Surgery Research Center, University of Insubria \\ (Varese-Como), Varese, Italy \\ ${ }^{3}$ Professor and Chairman, Department of Endocrine Surgery, Hospital del Mar, Barcelona, Spain \\ ${ }^{4}$ Associate Professor, Department of Endocrine Surgery, 3rd Chair of General Surgery, Jagiellonian \\ University Medical College, Krakow, Poland \\ ${ }^{5}$ Professor and Chief, Department of General Surgery and Surgical Oncology, MacLean Center for Clinical Medical Ethics \\ University of Chicago, Chicago, USA \\ ${ }^{6}$ Professor and Chairman, Department of General, Visceral and Vascular Surgery, Martin Luther University \\ Halle-Wittenberg, Halle, Germany \\ ${ }^{7}$ Massachusetts Eye and Ear Infirmary Division of Thyroid and Parathyroid Surgery, Research Assistant, Department of \\ Thyroid and Parathyroid Surgery, Massachusetts Eye and Ear Infirmary, Harvard Medical School, Boston, USA \\ Endocrine Surgery Service-Surgical Oncology, Massachusetts General Hospital \\ 243 Charles Street, Boston, Massachusetts, USA \\ ${ }^{8}$ Associate Professor of Otology and Laryngology, Department of Thyroid and Parathyroid Surgery, Massachusetts Eye and \\ Ear Infirmary Harvard Medical School, Boston, USA, Endocrine Surgery Service-Surgical Oncology \\ Massachusetts General Hospital, 243 Charles Street, Boston, Massachusetts 02114, USA
}

Correspondence: Manuel C Durán Poveda, Professor, Department of Endocrine Surgery, University Hospital of Fuenlabrada Health Sciences School, King Juan Carlos University, Camino del Molino $\mathrm{n}^{\circ}$ 2, 28942 Fuenlabrada, Madrid, Spain e-mail: mduran.hflr@salud.madrid.org

\section{ABSTRACT}

One of the most feared complications in thyroid surgery is injury to the superior laryngeal nerve or recurrent laryngeal nerve. Neural identification during surgery is insufficient to assess nerve injury. Intraoperative nerve monitoring of the vagal nerve and recurrent laryngeal nerve during thyroid surgery is a new adjunct designed to allow better identification of nerves at risk and therefore reduce complications related to their injury. This new working tool does not substitute adequate surgical technique but merely provides the surgeon with an adjunct to routine visual identification and functional assessment. The use of nerve monitoring requires standardization of the monitoring procedure. Pursuant to this, we will discuss in two related articles the current state of the art standardized technique of nerve monitoring in thyroid surgery. The aim of part 1 is to provide a concise overview of nerve monitoring in thyroid surgery and its effectiveness. This will include a brief review of the surgical anatomy of the recurrent laryngeal nerve and the key landmarks used to identify the nerve during surgery. Part 2 will describe how to perform the standardized nerve monitoring in a step by step fashion during thyroid surgery which will diminish variable results and misleading information associated with a nonstandardized nerve monitoring procedure.

Keywords: Neuromonitoring, Vagus nerve, Recurrent laryngeal nerve, Thyroid surgery.

\section{INTRODUCTION}

Anatomic and functional preservation of the recurrent laryngeal nerve (RLN) is the gold standard in thyroid surgery. Visual identification of RLN has decreased the rates of permanent RLN palsy during thyroid and parathyroid operations. However, unexpected RLN palsy still occurs. This is one of the most frequent causes of medicolegal litigation after thyroid and parathyroid surgery. In addition, most nerve injuries are not recognized intraoperatively and visualization of the nerve is insufficient to assess nerve damage. Intraoperative neuromonitoring (IONM) of the RLN represents an adjunct to routine visual identification of the nerve during surgery and provides a broader vision of surgical anatomy incorporating new clinical neurophysiologic and functional patterns to surgical practice.

IONM is not intended as a substitute for adequate surgical technique. The identification of neural structures during surgery of the neck, however, can be difficult even with extensive anatomical knowledge and surgical experience. RLN monitoring during thyroidectomy facilitates anatomic neural identification and dissection in order to avoid iatrogenic injuries, helps in 
resident medical education and training and gives a prognostic value regarding postoperative neural function. ${ }^{1-3}$ Although these three specific functions are sufficient to consider the value of IONM as a new technology that allows a refinement in surgical technique and its outcome, RLN monitoring's main role is based on the ability of predicting intraoperatively postoperative glottic function. Therefore, the use of IONM may also facilitate intraoperative decision-making for bilateral thyroid surgery.

In the last 10 years, noninvasive RLN monitoring has been introduced in Europe in thyroid and parathyroid surgery. ${ }^{4-12}$ Recently, Sturgeon et al published an internet-based survey among members of the American Association of Endocrine Surgeons regarding the different attitudes, usage patterns and predictors of use of IONM in their clinical practice. They found that a $37.1 \%$ of the respondents did use IONM; this category was divided into routine (13.8\%) and selective users (23.3\%) of nerve monitoring. ${ }^{13}$ Recent studies have pointed out the benefit of nerve monitoring for younger surgeons and low volume thyroid surgeons. Indeed, some studies have suggested an increase in surgeon confidence among users of neuromonitoring. ${ }^{13-16}$

The lack of standardized procedures for nerve monitoring during thyroid and parathyroid operations has led to variable and disparate results. A review of the relevant medical literature on RLN monitoring over the last 10 years shows that IONM was performed in an unstandardized way and, thus, it is difficult to compare studies and obtain a concurred opinion on IONM. ${ }^{1,5,8,10-12,16-25}$ Although these studies do have varying specific conclusions almost all agree that RLN monitoring can be effective in assisting the surgeon in the identification of the nerve, especially during revision surgery where the anatomic course can be distorted, and in predicting nerve functional integrity. Recently, a standardized approach to IONM has been established by Randolph et al. ${ }^{26}$

\section{RECURRENT LARYNGEAL NERVE MONITORING-STATE OF THE ART}

Meticulous technique during thyroidectomy with anatomic and functional preservation of the RLN is critical to ensure the success of thyroidectomy. The morbidity of the procedure remains a concern for endocrine surgeons. Nerve injury can be a very significant and debilitating complication for patients with a clear impact on their quality of voice especially in professional voice users. The wide spectrum of clinical findings in RLN injury vary from no discernible effect, minor volume reduction of the voice to full blown paralytic dysphonia and dysphagia associated with aspiration that can lead in severe cases to significant pulmonary infectious complications.

Voice dysfunction after thyroid surgery may be caused by many factors. ${ }^{27,28}$ Changes in voice after thyroid surgery are usually caused by injury to the superior laryngeal nerve and/or RLN. These voice changes can be measured and studied in order to quantify the degree of nerve injury. 1,29-31

The frequency of permanent RLN paralysis in surgeons who treat a large number of patients has been reported less than $2 \% .{ }^{15,23,32}$ There is a tendency to believe that the rates of permanent RLN paralysis reported in the literature have been underestimated for several reasons. First, endocrine units with a short surgical experience in thyroid and parathyroid surgery and unfavourable data are less likely to report their results. Second, most injuries are not detected intraoperatively by surgeons and in some studies, only up to $10 \%$ of lesions are evident during surgery and third, not all the patients undergo postoperative laryngeal examination. ${ }^{33-36}$

Most surgeons attempt to identify the RLN as a priority during surgery to minimize the rate of complications. ${ }^{37,38}$ Routine intraoperative identification of the RLN is associated with a lower incidence of RLN injury in terms of transient paralysis (3\%) and permanent paralysis (1\%) compared with the nonidentification of the nerve (transient paralysis of 8-9\% and permanent of 5\%) according to different series. ${ }^{16,25,39}$ Therefore, it is essential that the nerve is identified and dissected out along its course in all cases. ${ }^{29,37,40}$

\section{RLN Anatomy}

There is some variation in the origin, course and relationships of the RLN between the left and right sides. RLN, as a branch of the vagus, originates from the latter at the level of the arch of the aorta on the left and the right subclavian artery on the right. It is from this point that they turn superiorly and ascend in the tracheoesophageal groove as the right and left recurrent laryngeal nerves to enter the larynx ${ }^{21}$ (Fig. 1).

The right vagus descends in the neck and at the level of the right subclavian artery loops around it giving the right RLN which curves up and behind the subclavian artery ascending in the neck to innervate the larynx. The right RLN enters the neck

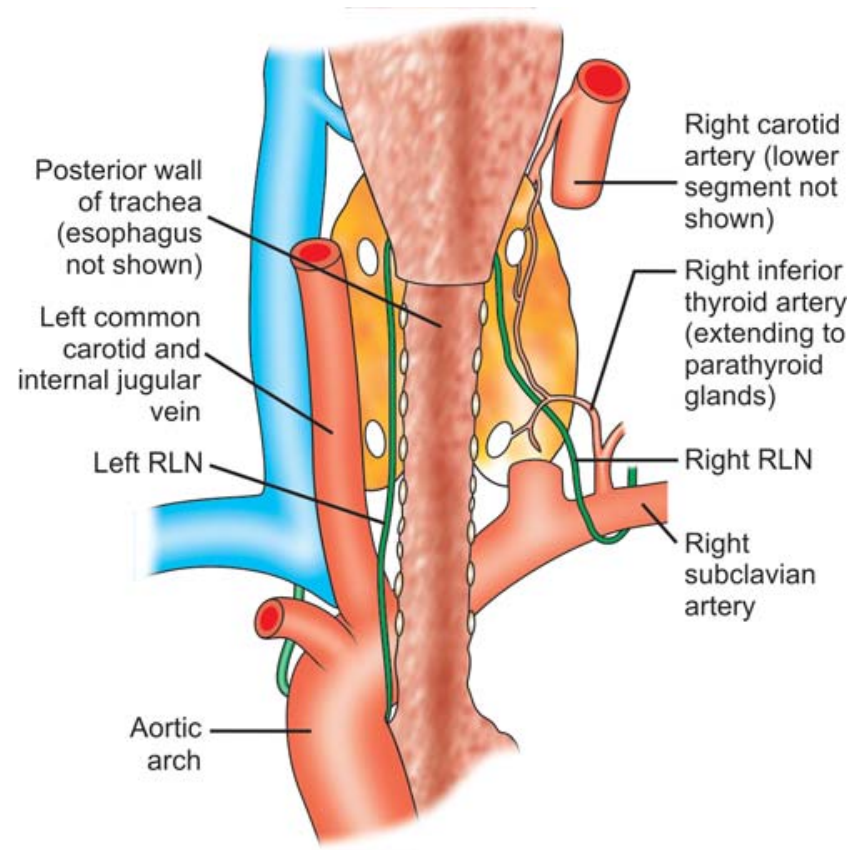

Fig. 1: Anatomy of the thyroid region. Recurrent laryngeal nerve course in the upper chest and neck (posterior view). Note that the relationships of both nerves with different anatomical landmarks [Courtesy: Reproduced with permission from Randolph GW. Surgical anatomy of the recurrent laryngeal nerve. In: Randolph GW (Ed). Surgery of the thyroid and parathyroid glands. Philadelphia, PA: Saunders 2003] 
base running more laterally to the tracheoesophageal groove than the left RLN. In approximately 0.5 to $1 \%$ of cases, an anomalous nonrecurrent RLN has been reported originating from the cervical portion of the vagus nerve in association with a vascular anomaly during the embryonic development of the aortic arches leading to a right subclavian artery takeoff from the distal aortic arch (arteria lusoria). ${ }^{37}$

The left RLN arises from the vagus nerve underneath the aortic arch and enters the thoracic inlet from the superior mediastinum in a more paratracheal position. From this point, it runs superiorly near the tracheoesophageal groove in close relationship with distal branches of the left inferior thyroid artery, giving off neural branches to surrounding structures, the trachea, esophagus and heart, before extending underneath the fibers of the inferior constrictor muscle to innervate the larynx. ${ }^{37}$

The RLN exhibits some variability in its course regarding its topographical location and requires special attention by the thyroid surgeon to prevent damage to the nerve during thyroidectomy. Three landmarks have been proposed to identify the RLN during surgery: The inferior thyroid artery, the tracheoesophageal groove and the RLN laryngeal entry point.

The relationship of the RLN with the inferior thyroid artery is the most frequent examined landmark during thyroid and parathyroid surgery. ${ }^{21}$ The artery emerges laterally to the nerve and then displays a medial course, extending to the thyroid with an inconstant relationship with the RLN, showing up to 28 different RLN inferior thyroid artery anatomical patterns in some studies. ${ }^{37,41,42}$ However, a common relationship is that the artery and nerve intersect; the nerve runs in between the terminal branches of the artery. These frequent variations confer a special surgical vulnerability for the nerve at this level.

The course of the RLN through the tracheoesophageal groove is not entirely reliable as a constant surgical landmark. The distance from the trachea and oesophagus varies from side to side. The localization of the RLN as it courses into the tracheoesophageal groove is more variable on the right side than on the left, with the left RLN entering from below the aortic arch in a more paratracheal position and extending upward in or near the tracheoesophageal groove in almost 60 to $70 \%$ of the cases, whereas the right RLN is located in that position only in $59 \%$ of cases. ${ }^{43}$

The ligament of Berry or posterior suspensory ligament of the thyroid is a fibrous condensation of the thyroid capsule located on either sides of the trachea that anchors the thyroid to the laryngotracheal complex. This ligament contains terminal branches of the inferior thyroid artery and can be very closely associated with adjacent thyroid tissue. The RLN has a close relationship with the suspensory ligament of Berry and courses through it in 25 to $30 \%$ of cases or passes deep to the ligament $75 \%$ of the time. ${ }^{43}$

The posterior process or tubercle of Zuckerkandl, an embryological remnant of thyroid tissue located at the posterolateral margin of the thyroid lobe caudal to the ligament of Berry, when present, has a varying relationship to the RLN and can modify its distal course making dissection of the RLN at this point more difficult, particularly when it enlarges in patients with goiter. ${ }^{44}$ The tubercle of Zuckerkandl may be absent or not recognized in some patients during surgery so it cannot be a reliable marker for the RLN. ${ }^{21}$ The nerve is at greatest risk for injury at this posterior side of the thyroid where it lies extremely close to the thyroid capsule and can angulate before entering the larynx at the laryngeal entry point. Randolph identifies the laryngeal entry point as the point of disappearance of the RLN under the lowest fibers of the inferior constrictor (Fig. 2). ${ }^{37}$ The inferior edge of the thyroid cartilage (inferior cornu) represents a palpable landmark indicating the laryngeal entry point of the RLN. The RLN entry point is the most reliable landmark for the identification of the RLN in the neck. ${ }^{37,45}$

It is important to keep in mind during thyroid surgery the potential of extralaryngeal RLN branching. Extralaryngeal branching is a very common event with a reported incidence in literature of 20 to $95 \% .{ }^{21}$ In 30 to $78 \%$ of cases, extralaryngeal RLN branches are displayed before the point where the nerve enters the larynx (laryngeal entry point) posterior to the cricothyroid muscle and above the inferior thyroid artery. About $30 \%$ of these RLN branching are considered true RLN extralaryngeal branches (i.e. with positive laryngeal electromyographic activity) and the absence of identification during surgery puts them at serious risk for injury. ${ }^{37,46-48}$ Careful dissection and exposure of the RLN and branches are mandatory to avoid inadvertent injury. ${ }^{49}$

Sancho et al assessed the impact of surgical injury to the extralaryngeal branching of the RLN on vocal cord dysfunction. They concluded that branched nerves suffered more injuries during surgical interventions and were more likely to be associated with vocal cord dysfunction than nonbranched RLNs. $^{50}$

The RLN is most prone to be injured in the last $2 \mathrm{~cm}$ of its course. Certain circumstances such as large substernal goiters,

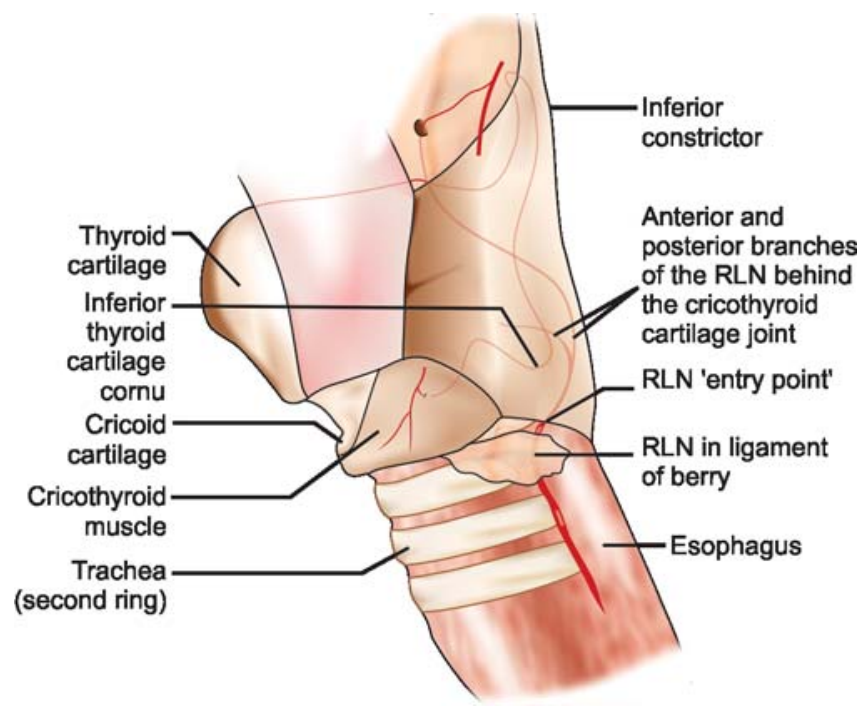

Fig. 2: Side view of the larynx showing the ligament of Berry, the relationship of the recurrent laryngeal nerve with the ligament of Berry and the laryngeal entry point [Courtesy: Reproduced with permission from Randolph GW. Surgical anatomy of the recurrent laryngeal nerve. In: Randolph GW (Ed). Surgery of the thyroid and parathyroid glands. Philadelphia, PA: Saunders 2003] 
Graves’s disease, thyroiditis, thyroid cancer, revision surgery or previous radiation therapy may distort anatomical relationships and can make identification of the RLN difficult (Table 1). 2,8,10,20,21,28,51-54

\section{RLN Monitoring}

RLN monitoring represents a strong support to routine visual identification of the nerve during surgery. IONM has been claimed in numerous studies to reduce rates of nerve injury during thyroid surgery compared with anatomical dissection and visual identification.,55

From a practical point of view, there are different ways of identifying the RLN. ${ }^{20,21,37,55}$

1. Visual identification: The gold standard for RLN preservation during surgery is routine visual identification of the nerve. The RLN is identified as a whitish structure of approximately 2 to $3 \mathrm{~mm}$ width with its vasa nervorum running on its surface (Fig. 3). This appearance can be modified in situations where the nerve is placed on stretch such as in the presence of cancer or a large goitre. IONM confirms a surgeon's visual impression and nerve electromyographic activity available with RLN monitoring adds functional information to visual identification alone.

Table 1: Clinical situations at high-risk/higher consequence of RLN injury

- Lack of RLN identification during surgery

- Bilateral surgery

- Surgery for substernal goiter

- Surgery for Graves' disease and thyroiditis

- Reoperative thyroid and parathyroid surgery where all anatomical landmarks are often distorted

- Surgery for cancer including important lymph node dissectionresection

- Surgery on an only functioning RLN

- Surgery after external beam radiation

- Surgery associated with longer operating room time and patients brought back to surgery because of bleeding

- Anatomic variations of the RLN including anomalous courses outside the tracheoesophageal groove, distorted relationships with the inferior thyroid artery, tubercle of Zuckerkandl or Berry's ligament, branching at some distance from the larynx and the existence of a nonrecurrent RLN

- Surgeons with little experience in thyroid-parathyroid surgery.

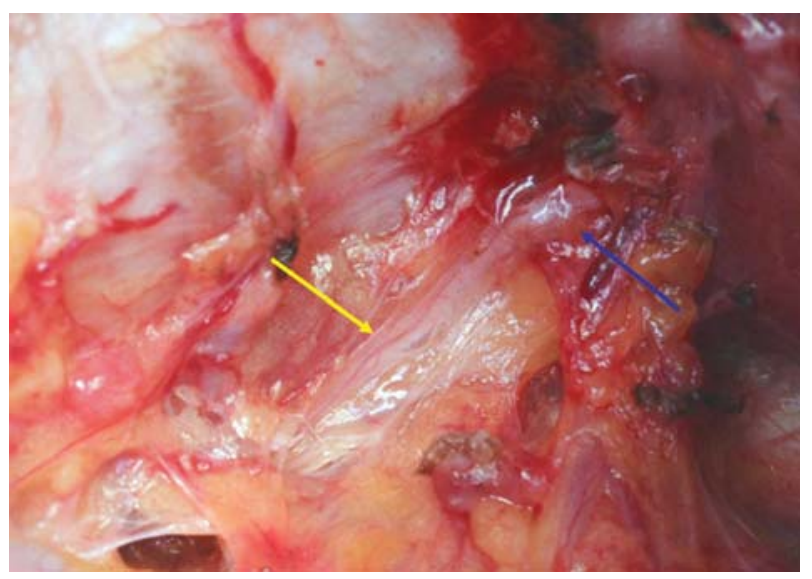

Fig. 3: Recurrent laryngeal nerve with its vasa vasorum (yellow arrow) and laryngeal entry point (blue arrow)
2. Palpation: Palpation of the nerve has been suggested as a technique for nerve identification. This method involves upward retraction of the partially mobilized lobe and palpation of the nerve through the connective tissue located around and below the inferior thyroid pole against the trachea. Given the obvious potential for stretch injury through such manipulation is not recommended.

3. Intraoperative neuromonitoring (IONM): Currently, intraoperative nerve monitoring is fully incorporated into surgical practice in some specialties, such as otolaryngology, neurosurgery, plastic surgery, maxillofacial surgery and more recently in general surgery. ${ }^{15,31,56-59}$

The first reports of early IONM technologies used to reduce the risk to RLN were published by Shedd and Durham in 1965. Previously, Basmajian described transcutaneous intramuscular vocal cord electrodes and later in 1970, Flisburg and Lindholm inserted a needle through the cricothyroid membrane into the vocalis muscle for neuromonitoring of the inferior laryngeal nerve during thyroid surgery. ${ }^{21,37,60}$ Many advances have occurred in the field of neurophysiology and nerve monitoring since this time. . $^{2,15,20,61}$

IONM allows both the superior and inferior laryngeal nerves to be reliably identified during surgery. It is also an important adjunct in dissection, adding to visual identification and provides functional neural prognosis. ${ }^{3,8,20}$ RLN monitoring's main function is that of intraoperative prediction of postoperative function.

\section{Effectiveness of RLN Monitoring}

Nerve monitoring is very useful in the following situations: ${ }^{6,37,59}$

1. RLN identification: The nerve is located and mapped out in the surgical field through stimulation (neural mapping). Directed dissection of the stimulated area allows visualization of the nerve. Multiple studies have found neuromonitoring useful for identifying the nerve with success rates between 98 and $100 \% .{ }^{62}$ IONM is a noninvasive method that enables early identification of the nerve especially useful in patients with anatomic variants allowing the surgeon to identify potentially nerves at risk. ${ }^{21,54}$ RLN monitoring is a more reliable surgical tool to assess nerve injury than visual inspection alone.

2. Aid in nerve dissection: Intraoperative RLN stimulation is an additional safety tool which is very useful in cases of difficult dissection or anatomical variations of the nerve. Once the nerve is identified, we proceed to electrical stimulation. Every structure that mimics a nerve can be stimulated in the surgical field. Intermittent stimulation of the dissected field allows tracing the nerve and its branches. ${ }^{49}$ IONM may assist in differentiating nerve from nearby nonnervous tissue, especially useful in heavily scarred tissue such as reoperations.

3. Predicting postoperative vocal cord function and identification of nerve injury site: RLN monitoring's most important function is that of intraoperative prediction of 
postoperative vocal cord function. Several studies have revealed the limited ability of the surgeon to judge a RLN injury intraoperatively. The limited data available in the literature suggests that only approximately $10 \%$ of nerve injuries are detected visually intraoperatively by surgeons. Compression, crushing, thermal injury, ischemia, ligature, stretch or traction can block the neural conduction (neuropraxia) without anatomical interruption of the RLN. ${ }^{1,21,63}$

IONM is also used to predict vocal cord function and elucidate the mechanism of nerve injury. Nonstandard application of IONM techniques can lead to important misleading information and conflicting results. A recent review of IONM in the literature using evidence-based criteria by Dralle et al revealed high negative predictive values (92 to $100 \%$ ) when comparing IONM findings with their corresponding postoperative glottic observation (postoperative cord function prediction). ${ }^{8}$ Based on these studies, patients with an intact electromyographical signal after surgery (negative IONM test or preserved nerve function) would generally have a normal vocal cord function. The results of loss of signal (LOS) in these studies however were associated with a wide range of postoperative result ranging from normal vocal cord function to transient or permanent cord palsy. Following this intraoperative LOS, consistent LOS evaluation algorithms would be expected to strengthen positive predictive values. Standards should be applied to IONM to facilitate uniform comparable and accurate neural monitoring.

Conversely, when using audio-only nerve locators, several studies have shown highly variable positive predictive values $(10-90 \%)^{6,17,22,64}$

If an abnormal or absent IONM signal is evidenced during bilateral surgery, the policy of continuing surgery or the strategy to deal with the contralateral side can be reviewed in order to avoid potential bilateral vocal cord paralysis and tracheotomy.

Electrophysiologic RLN integrity at the end of surgery does not always correlate with normal postoperative vocal fold mobility. In these cases, electrophysiologic monitoring of the RLN integrity may be more predictive if vagus nerve is stimulated rather than RLN. An injury far from the larynx may be missed if the surgeons test the nerve by stimulating the uninjured region of the nerve distally. Vagus stimulation ensures the entire neural circuit is intact. 6,9,24,65-69

A basic principle when using RLN monitoring is that the surgeon must see the nerve and get electrical confirmation before cutting any tissue. A standardized technique of noninvasive RLN monitoring includes both vagal and RLNs stimulation before, during and after thyroid resection. No real consensus has been yet established about which stimulation intensity level (intensity of electrical current) should be used to obtain a safe and optimal electromyographic response. Usually, 1 to 2 milliamps is more than sufficient. One milliamp if directly applied on a dissected nerve and 2 milliamps if mapping or monitoring in a not fully dissected field. Nevertheless, multiple studies have demonstrated the safety of repetitive nerve stimulation and the absence of ill effects in children and adults during thyroidectomy. ${ }^{30,37,70-72}$ Repetitive vagus stimulation is well tolerated and is not associated with respiratory or cardiovascular effects.

The minimum requirements for optimal use of nerve monitoring include:

1. Preoperative laryngoscopy: Laryngoscopic examination of glottic motion is essential to asses the functional status of the vocal cords prior to the beginning of nerve monitoring. Information obtained from glottis examination allows comparison of preoperative clinical findings with intraoperative electrophysiological signals derived from nerve stimulation. Therefore, preoperative glottic observation is mandatory in all cases.

2. Presurgical testing of vagus nerve: Routine stimulation of vagus nerve as the first surgical step before identification of the RLN guarantees that RLN neuromonitoring system is functioning correctly.

3. Postsurgical testing of vagus nerve: Final testing of vagus nerve at the completion of the operation shows a rigorous testing of postoperative glottis function with an added prognostic value and ensures the entire RLN is intact. This final step of the procedure allows for testing of the entire neural circuit.

4. Postoperative glottic observation: Early postoperative laryngoscopy to check vocal cord mobility is of paramount importance and highly recommended in all patients. Postoperative glottic examination is necessary in all cases in order to have a functional registry of the larynx after surgery. It is essential to improve the prognostic correlation between end of surgery neural stimulation and postoperative glottic function. The need for rigorous and concise postoperative glottic function information in all cases is essential for accurate monitoring. ${ }^{23,73}$

\section{SUMMARY}

The RLN has a variable anatomic course that makes it vulnerable when performing thyroid and parathyroid surgery. Iatrogenic injury to the RLN remains a serious complication after thyroid surgery and it is a leading reason for medicolegal litigation. ${ }^{74}$ Surgical exposure and visual identification of the RLN represent the gold standard in thyroid surgery and have been reported to be associated with lower rates of nerve injury.

Intraoperative neuromonitoring is an important adjunct in RLN dissection and functional neural prognosis. Standardized IONM procedures help to obtain accurate monitoring information which has application in initial neural mapping, subsequent neural dissection and postoperative neural prognostication.

\section{ACKNOWLEDGMENT}

This study was supported in part by a grant from the Spanish Ministry of Science and Innovation (Fondo de Investigaciones Sanitarias, PI09/90654:

"Evaluación Clínica de un Sistema de Monitorización Nerviosa Intraoperatoria para evitar la lesión de los nervios 
laríngeos en la Cirugía Tiroidea”. Instituto de Salud Carlos III. Ministerio de Ciencia e Innovación. España).

\section{REFERENCES}

1. Witt RL. Recurrent laryngeal nerve electrophysiologic monitoring in thyroid surgery: The standard of care? J Voice 2005;19:497-500.

2. Dionigi G, Rovera F, Carrafiello G, Bacuzzi A, Boni L, Dionigi R. New technologies in ambulatory thyroid surgery. Int J Surg 2008;6:S22-25.

3. Dionigi G, Barczynski M, Chiang FY, Dralle H, DuranPoveda $\mathrm{M}$, Iacobone $\mathrm{M}$, et al. Why monitor the recurrent laryngeal nerve in thyroid surgery? J Endocrinol Invest 2010;33: 819-22.

4. Dralle H. Intraoperative neuromonitoring in thyroid surgery and surgery of the parathyroid gland. Zentralb Chir 2002;127: 393-94.

5. Thomusch O, Sekulla C, Walls G, Dralle H. Intraoperative neuromonitoring of surgery for benign goiter. AM J Surg 2002;183:673-78.

6. Dralle H, Sekulla C, Haerting J, Timmermann W, Neumann HJ, Kruse E, et al. Risk factors of paralysis and functional outcome after recurrent laryngeal nerve monitoring in thyroid surgery. Surgery 2004;136: 1310-22.

7. Moroni E, Jonas J, Cavallaro A, Sapienza P, MC, Bahr R. Intraoperative neuromonitoring of the recurrent laryngeal nerve. Experience of 1000 consecutive patients. G Chir 2007;28: 29-34.

8. Dralle H, Sekulla C, Lorenz K, Brauckhoff M, Machens A. German IONM Study Group. Intraoperative monitoring of the recurrent laryngeal nerve in thyroid surgery. World J Surg 2008;32:1358-66.

9. Dionigi G, Bacuzzi A, Boni L, Rovera F, Dionigi R. What is the learning curve for intraoperative neuromonitoring in thyroid surgery? International Journal of Surgery. Int J Surg 2008;6: S7-12.

10. Cavicchi O, Caliceti U, Fernández IJ, Macrì G, Di Lieto C, Marcantoni A, et al. The value of neurostimulation and intraoperative nerve monitoring of inferior laryngeal nerve in thyroid surgery. Otolaryngol Head Neck Surg 2009;140: 866-70.

11. Goretzki PE, Schwarz K, Brinkmann J, Wirowski B, Lammers BJ. The impact of intraoperative neuromonitoring (IONM) on surgical strategy in bilateral thyroid diseases. Is it worth the effort? World J Surg 2010;34:1274-84.

12. Atallah I, Dupret A, Carpentier AS, Weingertner AS, Volkmar PP, Rodier JF. Role of intraoperative neuromonitoring of the recurrent laryngeal nerve in high-risk thyroid surgery. J Otolaryngol Head Neck Surg 2009;38:613-18.

13. Sturgeon C, Sturgeon T, Angelos P. Neuromonitoring in thyroid surgery: Attitudes, usage patterns, and predictors of use among endocrine surgeons. World J Surg 2009;33:417-25.

14. Hemmerling T, Schmidt J, Bosert C, Jacobi KE, Klein P. Intraoperative monitoring of the recurrent laryngeal nerve in 151 consecutive patients undergoing thyroid surgery. Anesth Analg 2001;93:396-99.

15. Becker PM, Gourin CG. New technologies in thyroid surgery. Surg Oncol Clin N Am 2008;17:233-48.

16. Jonas J, Bähr R. Intraoperative neuromonitoring of the recurrent laryngeal nerve-results and learning curve. Zentralbl Chir 2006;131:443-48.

17. Thomusch O, Sekulla C, Marchens A, Neumann HJ, Timmermann W, Dralle H. Validity of intraoperative neuromonitoring signals in thyroid surgery. Langenbecks Arch Surg 2004;389:499-503.
18. Timmermann W, Hamelmann WH, Thomusch O, Sekulla C, Grond S, Neumann HJ, et al. Effectiveness and results of intraoperative neuromonitoring in thyroid surgery. Statement of the interdisciplinary study group on intraoperative neuromonitoring of thyroid surgery. Chirurgia 2004;75: 916-22.

19. Loch-Wilkinson TJ, Stalberg PL, Sidhu SB, Sywak MS, Wilkinson JF, Delbridge LW. Nerve stimulation in thyroid surgery: Is it really useful? ANZ J Surg 2007;77:377-80.

20. Johnson S, Goldenberg D. Intraoperative monitoring of the recurrent laryngeal nerve during thyroid surgery revision. Otolaryngol Clin N Am 2008;41:1147-54.

21. Miller MC, Spiegel JR. Identification and monitoring of the recurrent laryngeal nerve during thyroidectomy. Surg Oncol Clin N Am 2008;17:121-44.

22. Barczyñski M, Konturek A, Cichoñ S. Randomized clinical trial of visualization versus neuromonitoring of recurrent laryngeal nerves during thyroidectomy. Br J Surg 2009;96:240-46.

23. Chiang FY, Lee KW, Chen HC, Chen HY, Lu IC, Kuo WR, et al. Standardization of intraoperative neuromonitoring of recurrent laryngeal nerve in thyroid operation. World J Surg 2010;34:223-29.

24. Chan WF, Lo CY. Pitfalls of intraoperative neuromonitoring for predicting postoperative recurrent laryngeal nerve function during thyroidectomy. World J Surg 2006;30:806-12.

25. Chiang FY, Lu IC, Kuo WR, Lee KW, Chang NC, Wu CW. The mechanism of recurrent laryngeal nerve injury during thyroid surgery. The application of intraoperative neuromonitoring. Surgery 2008;143:743-49.

26. Randolph GW, Dralle H (Eds). Abdullah H, Barczynski M, Bellantone R, Brauckhoff M, Carnaille B, Cherenko S, et al. In: International Intraoperative Monitoring Study Group. Electrophysiologic recurrent laryngeal nerve monitoring during thyroid and parathyroid surgery: International standards guideline statement. Laryngoscope 2011;121:S1-S16.

27. Hong KH, Kim YK. Phonatory characteristics of patients undergoing thyroidectomy without laryngeal nerve injury. Otolaryngol Head Neck Surg 1997;117:339-404.

28. McIvor NP, Flint DJ, Gillibrand J, Morton RP. Thyroid surgery and voice-related outcomes. Aust N Z J Surg 2000;70: 179-83.

29. Stojadinovic A, Shaha AR, Orlikoff RF, Nissan A, Kornak MF, Singh B, et al. Prospective functional voice assessment in patients undergoing thyroid surgery. Ann Surg 2002;236: 823-32.

30. Yarbrough DE, Farley DR. I can not sing anymore: Voice changes after thyroidectomy. Curr Surg 2003;60:554-60.

31. Lombardi CP, Raffaelli M, D’Alatri L, Marchese MR, Rigante M, Paludetti G, et al. Voice and swallowing changes after thyroidectomy in patients without inferior laryngeal nerve injuries. Surgery 2006;140:1026-1932.

32. Eisele DW. Intraoperative electrophysiologic monitoring of the recurrent laryngeal nerve. Laryngoscope 1996;106:443-49.

33. Lo CY, Kwok KF, Yuen PW. A prospective evaluation of recurrent laryngeal nerve paralysis during thyroidectomy. Arch Surg 2000;135:204-07.

34. Brennan J, Moore EJ, Shuler KJ. Prospective analysis of the efficacy of continuous intraoperative nerve monitoring during thyroidectomy, parathyroidectomy and paratidectomy. Otolaryngol Head Neck Surg 2001;124:537-43.

35. Steurer M, Passler C, Denk DM, Schneider B, Niederle B, Bigenzahn W. Advantages of RLN identification in thyroidectomy and parathyroidectomy and the importance of preoperative and postoperative laryngoscopic examination in more than 1000 nerves at risk. Laryngoscope 2002;112: 124-33. 
36. Chiang FY, Wang LF, Huang YF, Lee KW, Kuo WR. Recurrent laryngeal nerve palsy after thyroidectomy with routine identification of the recurrent laryngeal nerve. Surgery 2005;137:342-47.

37. Randolph GW. Surgical anatomy of the recurrent laryngeal nerve. In: Randolph GW (Ed). Surgery of the Thyroid and Parathyroid Glands. Philadelphia, PA: Saunders 2003:300-42.

38. Herranz-González J, Gavilán J, Matínez-Vidal J, Gavilán C. Complications following thyroid surgery. Arch Otolaryngol Head Neck Surg 1991;117:516-18.

39. Jatzko GR, Lisborg PH, Muller MG, Wette VM. Recurrent nerve palsy after thyroid operations-principal nerve identification and a literature review. Surgery 1994;115:139-44.

40. Djohan RS, Rodríguez HE, Connolly MM, Childers SJ, Braverman B, Podbielski FJ. Intraoperative monitoring of recurrent laryngeal nerve function. Am Surg 2000;66:595-97.

41. Sturniolo G, D'Alia C, Tonante A, Gagliano E, Taranto F, Lo Schiavo MG. The recurrent laryngeal nerve related to thyroid surgery. Am J Surg 1999;177:485-88.

42. Yalcxin B. Anatomic configurations of the recurrent laryngeal nerve and inferior thyroid artery. Surgery 2006;139:181-87.

43. Lore JM. Surgery of the thyroid gland. In: Tenta LT, Keyes GR, (Eds). Symposium on surgery of the thyroid and parathyroid glands. Vol 13. Piladelphia: WB Saunders 1980:69-83.

44. Gauger PG, Delbridge LW, Thompson NW, et al. Incidence and importance of the tubercle of Zuckerkandl in thyroid surgery. Eur J Surg 2001;167:249-54.

45. Kruse E, Olthoff A, Schiel R. Functional anatomy of the recurrent and superior laryngeal nerve. Langebeck's Arch Surg 2005;391:4-8.

46. Shindo ML, Wu JC, Park EE. Surgical anatomy of the recurrent laryngeal nerve revisited. Otolaryngol Head Neck Surg 2005;133:514-19.

47. Ardito G, Revelli L, D’Alatri C, Lerro V, Guidi ML, Ardito F. Revisited anatomy of the recurrent laryngeal nerves. AM J Surg 2004;187(2):249-53.

48. Schweizer V, Dörfl J. The anatomy of the inferior laryngeal nerve. Clin Otolaryngol 1997;22:362-69.

49. Barczynski M, Nowak W, Sancho JJ, Sitges-Serra A. The motor fibers of the recurrent laryngeal nerves are located in the anterior extralaryngeal branch. Ann Surg 2010;251:773-74.

50. Sancho JJ, Pascual-Damieta M, Pereira JA, Carrera $M^{\mathrm{a} J}$, Fontané J, Sitges-Serra A. Risk factors for transient vocal cord palsy after thyroidectomy. Br J Surg 2008;95:961-68.

51. Chan WF, Lang BH, Lo CY. The role of intraoperative neuromonitoring of recurrent laryngeal nerve during thyroidectomy: A comparative study on 1000 nerves at risk. Surgery 2006;140:866-72.

52. Myssiorek D. Recurrent laryngeal nerve paralysis: Anatomy and aetiology. Otolaryngol Clin North Am 2004;37:25-44.

53. Yarbrough DE, Thompson GB, Kasperbauer JL, Harper CM, Grant CS. Intraoperative electromyographic monitoring of recurrent laryngeal nerve in reoperative thyroid and parathyroid surgery. Surgery 2004;136:1107-15.

54. Horne SK, Gal TJ, Brennan JA. Prevalence and patterns of intraoperative nerve monitoring for thyroidectomy. Otolaryngology Head and Neck Surg 2007;136:952-56.

55. Randolph GW, Kobler JB, Wilkins J. Recurrent laryngeal nerve identification and assessment during thyroid surgery: Laryngeal palpation. World J Surg 2004;28:755-60.

56. Kartush JM, Bouchard KR (Eds). Neuromonitoring in otology and head and neck surgery. Raven Press, New York 1992.
57. Witt R. Facial nerve monitoring in parotid surgery: The standard of care? Otolaryngol Head Neck Surg 1998;119:468-70.

58. Harper CM. Intraoperative cranial nerve monitoring. Muscle Nerve 2004;29:339-51.

59. Edwards BM, Kileny PR. Intraoperative neurophysiologic monitoring: Indications and techniques for common procedures in otolaryngology, head and neck surgery. Otolaryngol Clin North Am 2005;38:631-42.

60. Flisburg K, Lindholm T. Electrical stimulation of the human recurrent laryngeal nerve during thyroid operation. Otolayngol Acta 1969;263:63-67.

61. Timon CI, Rafferty M. Nerve monitoring and thyroid surgery: Is it worthwhile? Clin Otolaryngol Allied Sci 1999;24:487-90.

62. Timmerman W, Hammelamnn W. Thyroid Surgery: Neuromonitoring of the RLN during thyroid surgery. Deutsches Arzteblatt 2004;101:7.

63. Marcus B, Edwards B, Yoo S, Byrne A, Gupta A, Kandrevas J, et al. Recurrent laryngeal nerve monitoring in thyroid and parathyroid surgery. The University of Michigan experience. Laryngoscope 2002;112:1594-61.

64. Tomoda C, Hirokawa Y, Uruno T, Takamura Y, Ito Y, Miya A, et al. Sensitivity and specificity of intraoperative recurrent laryngeal nerve stimulation test for predicting vocal cord palsy after thyroid surgery. World J Surg 2006;30:1230-33.

65. Kunath M, Marusch F, Horschig P, Gastinger I. The value of intraoperative neuromonitoring in thyroid surgeryA prospective observational study with 926 patients. Zentralbl Chir 2003;128:187-90.

66. Beldi G, Kinsbergen T, Schlumpf R. Evaluation of intraoperative recurrent nerve monitoring in thyroid surgery. World J Surg 2004;28:589-91.

67. Hermann M, Hellebart C, Freissmuth M. Neuromonitoring in thyroid surgery: Prospective evaluation of intraoperative electrophysiological responses for the prediction of recurrent laryngeal nerve injury. Ann Surg 2004;240:9-17.

68. Shindo M, Chheda NN. Incidence of vocal cord paralysis with and without recurrent laryngeal nerve monitoring during thyroidectomy. Arch Otolaryngol Head Neck Surg 2007;133:481-85.

69. Robertson ML, Steward DL, Gluckman JL, Welge J. Continuous laryngeal nerve integrity monitoring during thyroidectomy: Does it reduce risk of injury? Otolaryngol Head Neck Surg 2004;131:596-600.

70. Rea JL, Khan A. Clinical evoked electromyography for recurrent laryngeal nerve preservation: Use of an endotracheal tube electrode and postcricoid surface electrode. Laryngoscope 1998;108:1418-20.

71. Wu CW, Lu IC, Randolph GW, Kuo WR, Lee KW, Chen CL, et al. Investigation of optimal intensity and safety of electrical nerve stimulation during intraoperative neuromonitoring of the recurrent laryngeal nerve: A prospective porcine model. Head Neck 2010;32:1295-1301.

72. White WM, Randolph GW, Hartnick CJ, Cunningham MJ. Recurrent laryngeal nerve monitoring during thyroidectomy and related cervical procedures in the pediatric population. Arch Otolaryngol Head Neck Surg 2009;135:88-94.

73. Mihai R, Randolph, GW. Thyroid Surgery, voice and the laryngeal examination-time for increased awareness and accurate evaluation. World Journal of Endocrine Surgery 2009;1:1-5.

74. Angelos P. Recurrent laryngeal nerve monitoring: State of the art, ethical and legal issues. Surg Clin N Am 2009;89:1157-69. 